\title{
Lower Urinary Tract and Sexual Dysfunction in Neurological Patients
}

\author{
David B. Vodušek \\ Division of Neurology, University Medical Centre and Medical Faculty, University of Ljubljana, Ljubljana, Slovenia
}

\author{
Key Words \\ Neurogenic bladder - Lower urinary tract - Retention . \\ Incontinence - Sexuality · Erection · Orgasm · Lubrication . \\ Neurogenic dysfunction
}

\begin{abstract}
Background: Lower urinary tract dysfunction (LUTD) and sexual dysfunction (SD) are common in neurological patients due to a combination of lesions affecting relevant neural control, constraints imposed by sensorimotor and cognitive deficits and - particularly for SD - psychosocial consequences of chronic neurological disease. Summary: This review summarizes the etiology, diagnosis and treatment of LUTD and SD in neurological patients. Key Messages: LUTD may lead to serious health problems; both LUTD and SD significantly affect quality of life. Management of patients with spinal cord injury and dysraphism is undertaken in specialized centers according to established guidelines. Treatment of neurological patients with noncomplicated neurogenic LUTD or SD should preferentially be guided by a neurologist. Clinical Impact: For rational treatment of urinary symptoms, an accurate assessment is mandatory; the bladder and the sphincter need to be defined as normal, over- or underactive. Urodynamic testing is the gold standard for functional diagnosis; assessment of residual urine and uroflow are the minimal requirements before considering management. Dysfunction of desire, arousal and orgasm (ejaculation) may
\end{abstract}

be diagnosed by medical history and are amenable to counselling and treatment, which is - in the case of erectile dysfunction - evidence based. Further high-quality studies are necessary to test the best approaches for diagnosing and managing particular types of neurogenic LUTD and SD in the different neurological patient populations.

(c) 2014 S. Karger AG, Basel

\section{Introduction}

The field of neurourology arose in spinal cord injury centers, as the special needs of this patient population became recognized. Particularly after the Second World War, appropriate management of the 'neurogenic bladder' became widely established, thus contributing significantly to the survival of para/tetraplegics. Urodynamics became a powerful tool to demonstrate lower urinary tract (LUT) dysfunction (LUTD) and guide management strategies for the individual patient. It was also recognized that counselling on neurogenic sexual dysfunction (SD) was an important part of the rehabilitation of spinal cord injury patients. These conceptual and organizational achievements are only slowly spreading to the care of patients who were traditionally placed under the care of neurologists. Furthermore, there are few high-quality studies related to the epidemiology, appropriate diagnostics and therapy of neurogenic LUTD and neurogenic SD,

\section{KARGER}

E-Mail karger@karger.com

www.karger.com/ene
C 2014 S. Karger AG, Basel

0014-3022/14/0722-0109\$39.50/0
David B. Vodušek

Division of Neurology

University Medical Centre and Medical Faculty, University of Ljubljana

SI-1525 Ljubljana (Slovenia)

E-Mail david.vodusek@kclj.si 
but there is growing consensus that neurogenic pelvic organ dysfunction demands (also) neurological expertise. Clare J. Fowler, who established the first neurological unit devoted to neurogenic LUTD (and SD), thus coined for the discipline the term 'uroneurology'.

\section{Function and Neural Control}

Both the storage and elimination of urine, and sexual function (traditionally divided into desire, arousal, orgasm/ejaculation), are neurally controlled by coordinated activity of the autonomic and somatic nervous systems. In addition to the basic neural control of smooth and striated muscles involved in the functioning of organs dedicated to the above functions, both organ functions are set within complex behaviors dependent on brain control networks. These are increasingly being revealed by functional imaging.

Models of LUT neural control based on animal studies and functional imaging in humans have been proposed [1]. The two alternating functions of LUT (storage and elimination of urine), which are also appreciated through conscious sensation, are coordinated by a spinobulbospinal reflex. The periaqueductal gray is the sensory relay nucleus, and the pontine micturition center (PMC; in the dorsomedial pontine tegmentum) is the coordinator of efferent commands to the detrusor and the sphincters. Voluntary control is exerted by a complex brain network dominated by the lateral and medial prefrontal cortex (fig. 1). LUTD in patients can in principle be explained by the following model: lesions above the PMC produce an overactive bladder with coordinated sphincter; lesions involving the PMC or the spinal pathways to the detrusor and sphincter nuclei in the sacral spinal cord cause an overactive bladder with uncoordinated (dyssynergic) sphincter, and lesions to the peripheral sacral reflex arc cause an underactive bladder with underactive (denervated) sphincter.

In an individual patient, however, several factors define the particular state of the bladder and its outlet, and a 'neurological' classification of (neurogenic) LUTD has not proven clinically useful. The European Association of Urology Guidelines on Neurogenic Lower Urinary Tract Dysfunction [2] recommends the simple and therapeutically oriented Madersbacher classification system [3] for clinical practice, which differentiates between the state of the detrusor (on one side) and that of the urethral sphincter (on the other side) as either under- or overactive (or normal; fig. 2). The most common dysfunction related to

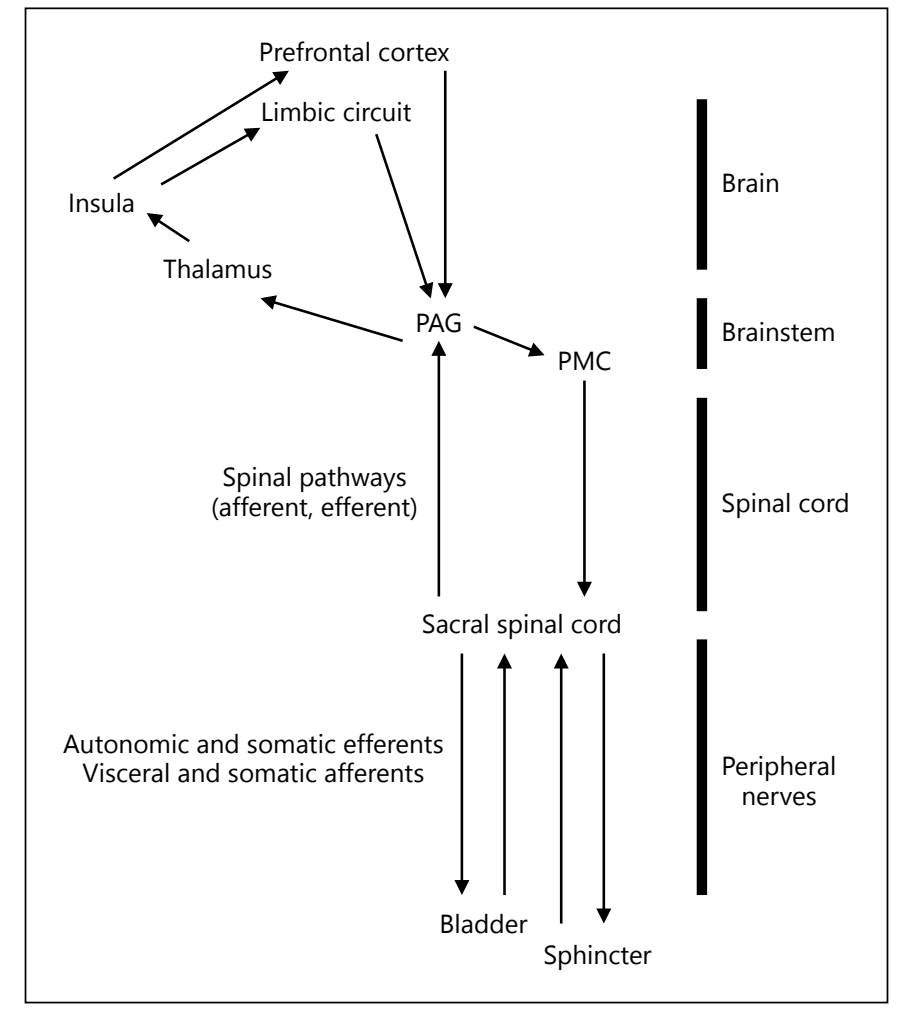

Fig. 1. A highly simplified model of neural control of the lower urinary tract. Afferent information is carried through visceral and somatic afferents to the spinal cord and to the periaqueductal gray (PAG) and then further on to several other brain areas. The prefrontal cortex exerts tonic inhibition on the 'brainstem switch' (periaqueductal gray and PMC). Receiving information on a full bladder, it decides on the social appropriateness of initiating voiding, coordinating the overall behavioral response. The limbic circuit (assessing emotional and safety homeostatic issues) may promote or inhibit 'voluntary' voiding. The periaqueductal gray is the relay center for both afferent information and downstream commands. The PMC coordinates synergic voiding by simultaneously activating the bladder and the inhibiting sphincter. Note that many nervous structures and areas and interactions have been omitted.

central nervous system lesions is an overactive detrusor with urgency, frequency, nocturia and urge incontinence; if associated with an overactive/dyssynergic urethral sphincter, the result is high intravesical pressure leading to kidney failure.

Sexual function is commonly understood as a sequence of desire (drive), excitation and orgasm (and resolution); however, this 'linear model' may not always accurately depict female sexuality [4]. Several cortical areas, particularly the temporal lobes, are involved in the sensorimotor, emotional and cognitive aspects of sexual behavior; the insula, anterior cingulate cortex and hypothala- 


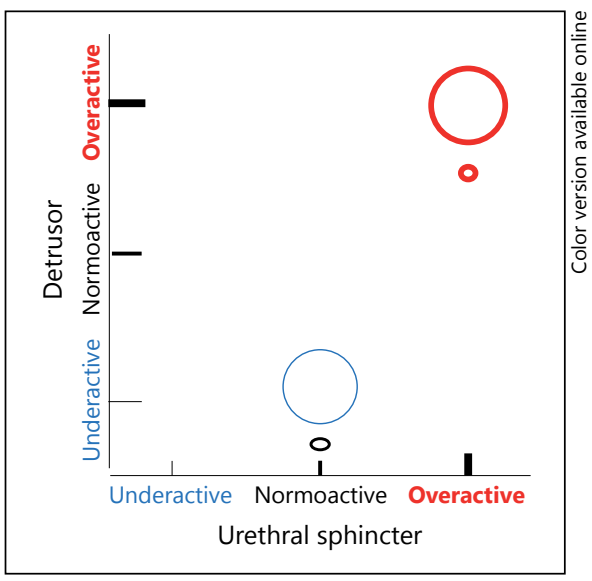

Fig. 2. The various patterns of neurogenic LUTD. While the normally functioning LUT is composed of a normoactive detrusor and normoactive urethral sphincter, either of these may become overactive or underactive as a consequence of neural system lesions. All different combinations of a normoactive, overactive or underactive detrusor with a normoactive, overactive or underactive urethral sphincter may be found in individual patients. This classification of LUTD was proposed by Madersbacher [3] and is clinically the most useful as it is directly applicable for planning rational treatment. Here, two common patterns of possible LUTD in two individual patients are shown, one demonstrating a combination of an underactive detrusor with a normoactive sphincter, the other an overactive detrusor with an overactive sphincter. The patient in the upper right corner is at risk of developing upper urinary tract involvement. The graph was constructed using the Madersbacher classification [3].

mus coordinate the activation of the autonomic nervous system. Facilitatory and inhibitory descending pathways modify the activity of thoracolumbar sympathetic, sacral parasympathetic and somatic spinal centers which control the sexual response, i.e. arousal, orgasm and ejaculation. Apart from ejaculation, the neural control of genital response is in principle the same for both genders; engorgement of genitals also occurs in women, but in fact it is the lubrication of the vagina which is the most relevant response and an equivalent to erection in the male (fig. 3). A normal hormonal milieu and vascular system, but also a healthy psychosocial context, are necessary for a fulfilled sexual life $[5,6]$.

The complexity of the prerequisites of normal sexuality frustrates attempts at simple correlations of SD with neurological lesions. For instance, although obviously brain areas are the substrate for desire and inducement of sexual arousal by noncontact stimulation, in chronic cauda equina patients there is not only erectile and ejaculatory dysfunction but also loss of desire [7].

LUT and Sexual Dysfunction in Neurological Patients

\section{Dysfunction in Neurological Disease}

Generally, LUTD and SD seem to be more common in neurological patients than in the general population; both may arise not only as a direct consequence of lesions but also as a result of therapy or motor, sensory and cognitive deficits. SD can also arise out of the psychosocial consequences of having a neurological disease. Apart from spinal cord injury patients, the data come from mostly small observational studies performed in often poorly defined patient populations. Therefore, data on epidemiology, symptomatology, urodynamic characteristics and the requirements for particular diagnostic procedures and appropriate therapy are only preliminary or based on expert opinion. In patients with spinal cord or cauda equina lesions, autonomic failure (including Multiple system atrophy (MSA)) and normotensive hydrocephalus, LUTD (and SD) are part of the clinical picture. In focal brain lesions, there are locations particularly related to LUTD (frontal) or SD (temporal). In multiple sclerosis (MS), Parkinson's disease and polyneuropathies, these dysfunctions typically appear with progression of the disease. Spinal and peripheral lesions may similarly affect both functions, but not brain disease; a case in point is epilepsy, which is - interictally - burdened solely with SD, contributing factors being therapy and psychosocial factors. Details of LUTD and SD symptoms in different diseases are beyond the scope of this short review. A thorough overview of this topic is provided by Fowler et al. [8].

In general, although a certain type of dysfunction may be 'typical' for a particular disease, in whole populations of patients, all possible LUTD and SD symptoms may appear (i.e. symptoms due to dysfunctional storage or dysfunctional elimination of urine; symptoms related to change in sexual desire, erection/lubrication, ejaculation/orgasm). SD commonly appears as loss of function, but 'hyperfunction' may also occur; this is most typically manifested as an increase in desire, as for instance in the context of dopaminergic treatment in Parkinson's disease.

LUTD has adverse effects on patient outcomes. It is accompanied by recurrent urinary tract infections. In spinal cord lesions and dysraphism, there is continuing danger of upper urinary tract involvement; however, this seems not to be common in neurological diseases such as MS and Parkinson's disease. LUT symptoms and urodynamic abnormalities worsen during the course of progressive neurological disease and even change from one symptom complex to another (from a storage problem into an elimination problem in MSA, for instance). 


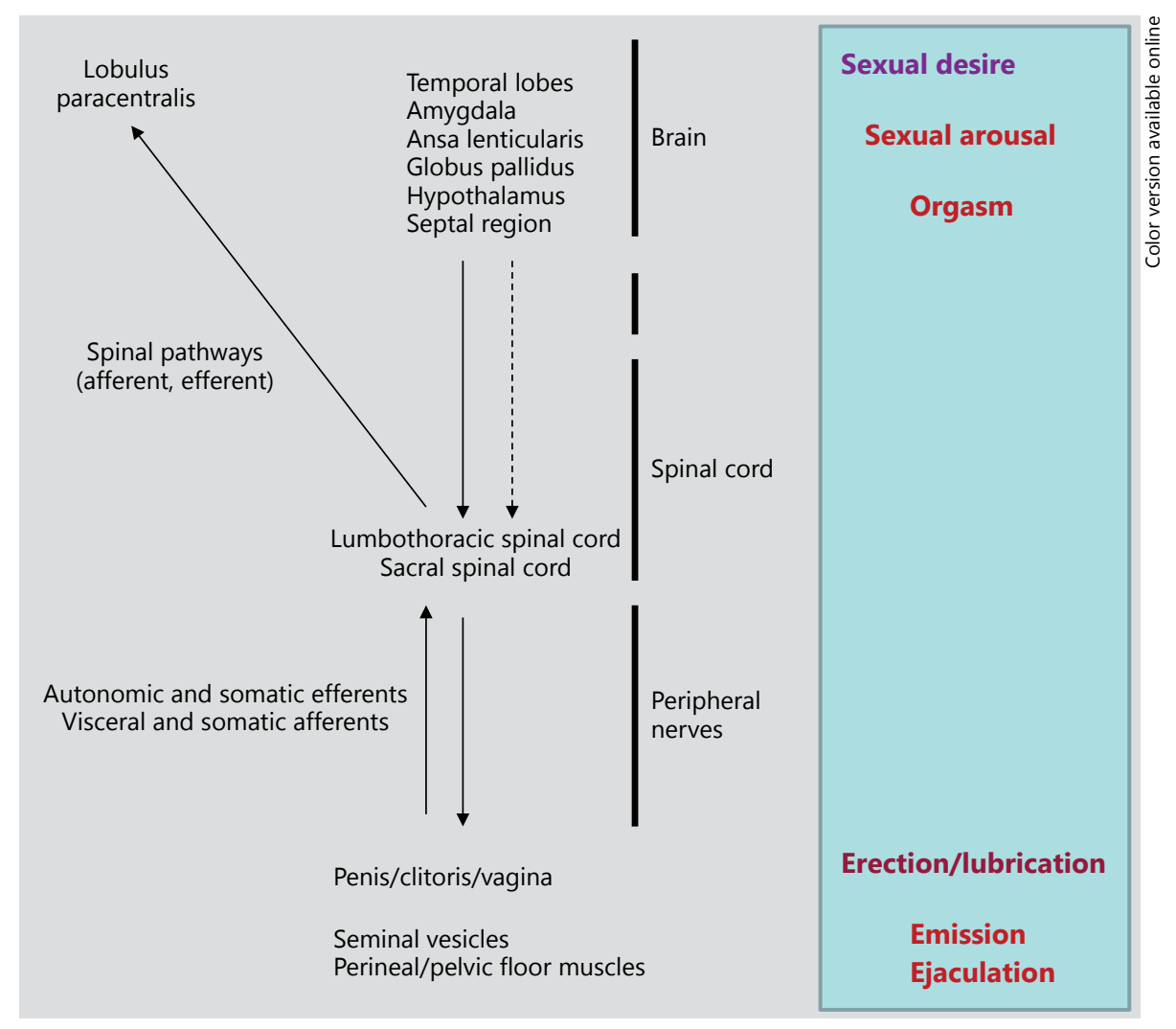

Fig. 3. A highly simplified model of neural control of sexual function. Primary sensory areas and inferior temporal lobes process sexual stimuli. The paracentral (parietal) lobule is the genital somatosensory area. Genital afferents may initiate the sexual response and reinforce it. The medial preoptic area integrates sensory and hormonal signals. The forebrain regulates the initiation and execution of sexual behaviour. The amygdala controls the reward aspects of sexual function. The paraventricular and paragigantocellularis nuclei project to the thoracic and lumbosacral nuclei, facilitating and inhibiting the sexual response, respectively. The sympathetic, parasympathetic and somatic efferents affect the sexual response. It is important to note that there is both a thora-

\section{Diagnostics}

Medical history, assessing LUT symptoms and keeping a bladder diary are the mainstay of a working diagnosis of LUTD. The use of a validated questionnaire is encouraged $[9,10]$. SD diagnosis is made by medical history alone (loss or increase of desire, anorgasmia, erectile dysfunction, priapism, ejaculation disorder, loss of lubrication, pain related to sexual organs or pain during sexual activity; however, patients often do not volunteer symptoms). Many questionnaires exist but are not necessary for routine practice [11]. As a rule, clinical examination of the neurological patient first seen complaining of LUTD or columbal-sympathetic as well as a sacral-parasympathetic pathway inducing sexual arousal in both genders. Orgasm including ejaculation activates the brain and thoracolumbal and sacral spinal cord and is affected by sympathetic and somatic efferents activating smooth muscle in the genitals and striated muscles of the pelvis in both genders. Overall, there are similarities in the brain control of sexual response between the genders. Of course, differences exist, for instance in the amount of brain activation in response to sexual imagery. Note that many nervous structures and areas, details of functional anatomy, interactions and differences between genders have been omitted for simplicity.
SD should include the anogenital region (assessment of anal sphincter tone and activation, eliciting the bulbocavernosus and anal reflex, sensation of touch and pinprick; the latter contributes the most towards the formulation of a neuroanatomical diagnosis). As for investigations, urodynamic testing is standard practice in patients with spinal cord injury and dysraphism. For patients with neurological disease and LUT symptoms, the practice of urological (urogynecological) consultation varies in different centers, but in all cases, 'structural lesions' should be ruled out. In many hospitals or even countries it is difficult to obtain neurourological counsel for a large number of heterogenous groups of neurological patients. It is suggested 
that basic clinical assessment (which should also assess the possibility of infection) should be performed by the neurological team, which is responsible for the long-term follow up of the patient. Because upper urinary tract involvement is rare in MS patients, it has been argued that urodynamic testing is not necessary for first-line symptomatic management of LUTD, though determination of postvoid residual urine should be performed. (In addition, infravesical obstruction needs to be excluded.) More invasive procedures are reserved for the patient with refractory problems [12]. The same can be argued for patients with brain lesions (i.e. stroke, Parkinson's disease). However, the International Consultation on Incontinence Consensus recommends that patients with suspected neurogenic LUTD should receive urodynamic tests (including filling and voiding cystometry). Videourodynamics is recommended if relevant anatomical abnormalities are expected [13]. It should be pointed out that no prospective study has investigated the cost benefit of invasive urodynamics versus simpler diagnostic techniques (assessment of postvoid residual urine, uroflow, upper urinary tract ultrasound). Single testing cannot provide guidance during long-term follow-up (in MS or movement disorder patients); urodynamic follow-up has been recommended. An assessment of residual urine using ultrasound by a specialized nurse on specific follow-up visits is practical, cheap and noninvasive.

Testing for testosterone in men with SD is only recommended if deficiency is suspected (in fact, erection is possible with abnormally low levels). Testing the genital response itself (erection, lubrication) is only performed for research purposes. If vascular insufficiency is suspected, an intrapenile injection of low-dose prostaglandin E will induce erection if the cause is psychological or neurological. This is an in-office procedure performed by a urologist.

Thus far, LUTD and SD have been discussed with respect to patients with a known neurological disease. A different issue is 'isolated' LUTD or SD presented to urologists who - not finding any 'urological' cause - suspect 'neurogenicity'. An isolated neurogenic SD case is most commonly due to polyneuropathy. Nevertheless, LUTD (and/or SD) may be early manifestations of some rare conditions (MSA, tethered cord syndrome, conus/cauda equina tumor). MS only rarely starts with LUTD (or SD). As a rule, a thorough history and clinical examination in such patients suffices, but occasionally appropriate imaging or - if a peripheral lesion is suspected - a concentricneedle EMG of the anal sphincter and bulbocavernosus reflex testing may be performed [14].

LUT and Sexual Dysfunction in Neurological Patients

\section{Therapy}

As a rule, management of patients with spinal cord injury and dysraphism is undertaken by neurourological/ neurorehabilitation teams in specialized centers according to established guidelines [15]. Treatment of other neurological patients with noncomplicated neurogenic LUTD or SD should be coordinated if not performed by the neurologist; a dedicated nurse in the team is also helpful, particularly for teaching intermittent (self) catheterization. Referral to urology is often impractical for firstline management and can be limited to complex patients or after first-line treatment fails. In the acute setting, use of a short-term indwelling catheter is safe. Urinary tract infections are common symptoms of chronic neurogenic LUTD, usually caused by incomplete emptying. Optimal treatment of urine retention is of primary importance. If urinary tract infections persist, a urologic evaluation is warranted in order to check for foreign bodies or bladder deformities (kidney and bladder stones are particularly common since certain types of bacteria may cause them). Chronic antibiotic use should be discouraged; this practice will almost certainly lead to infections with resistant bacteria. If antibiotics are warranted, use of intermittent or alternating courses of antibiotics (nitrofurantoin or trimethoprim) is recommended. In patients with indwelling catheters, asymptomatic bacteriuria should be expected and tolerated.

Scheduled voiding (bladder training) may be an adequate treatment for patients with mild symptoms of frequency and urgency or with hyposensitive bladders. Complete and regular emptying of the bladder may be all that is necessary to alleviate frequency, urgency, incontinence and bladder discomfort. In MS patients with difficulty in initiating voiding and poor bladder emptying, suprapubic vibration treatment has been reported to be effective [12]. Intermittent (self) catheterization should be introduced if the amount of the postvoid residual is above $100 \mathrm{ml}$. (This can be performed by the patient or caregiver. Motivation, cognition and adequate manual dexterity are necessary conditions). If symptoms of overactive bladder persist, an antimuscarinic drug should be added. The drug should be titrated individually; if one drug is not tolerated, another should be tried. Intermittent catheterizations may become necessary after introduction of the antimuscarinic agent because residual urine may be a side effect. In patients with bothersome day- or nighttime frequency, the synthetic analogue of vasopressin has been shown to confer symptomatic benefit, providing patients with up to $6 \mathrm{~h}$ of freedom from 
frequency. Desmopressin at a dose of 10-20 $\mu \mathrm{g}$ should only be used once in any 24-hour period; it should not be used regularly because of the danger of hyponatremia and it should not be used in patients over 65 years of age. Patients with resistant neurogenic bladder overactivity generally respond to botulinum toxin injections into the detrusor muscle. The beneficial effects of this therapy have a median duration of 9-11 months, but many patients need to start intermittent self-catheterization because of poor bladder emptying. Intrasphincteric injections of botulinum toxin may improve urinary retention and sphincter dyssynergia and ease catheterization. Electrical stimulation (ES) has been shown to inhibit the overactive detrusor (also in patients with neurogenic LUTD). 'Sacral neuromodulation' (sacral nerve stimulation with an implanted stimulator) has been the subject of the most controlled studies, but other approaches are also effective. Paradoxically, ES is also effective in treating retention. Overall, more studies are needed to define the role of ES in treating neurogenic LUTD. No drug treatment is available for neurogenic sphincter deficiency and detrusor underactivity.

In the highly disabled neurological patient with limited mobility, cognitive impairment, loss of manual dexterity and increased spasticity/rigidity, an indwelling catheter becomes a necessity. Traditionally, the suprapubic catheter is the preferred solution, but every use of indwelling catheters on a long-term basis causes complications, and controlled studies are limited. An indwelling catheter per se does not protect the upper urinary tract. Surgical solutions for some complicated neurogenic LUTD exist but should be practiced in expert neurourological centers. Further details on the management of LUTD according to consensus recommendations can be found in the report of Drake et al. [15].

Ideally, sexual education, counseling and specific suggestions regarding therapeutic methods should be offered to the patient with SD (and their partner) by the treating physician. The use of drug regimens should be discussed with the patient and corrected if necessary. In a limited number of patients, referral to a sexual therapist may be indicated. Methods for sexual rehabilitation in the context of neurologic disorders have been described [16], and specific suggestions for orgasmic/ejaculation disorders, poor lubrication and genital pain can be given $[17,18]$. Only neurogenic erectile dysfunction is further discussed here. If the problem is (partial) penile sensory loss, vibratory stimulation may help in producing a rigid erection sufficient for vaginal penetration. Sildenafil is an effective treatment for erectile dysfunction in men with MS, Parkinson's disease, spinal cord injury, spina bifida, diabetes, patients on dialysis and after renal transplantation and familial amyloidotic polyneuropathy (if desire is preserved). Overall, its efficacy in patients with an organic etiology of erectile dysfunction was reported to be $68 \%$, being lower in diabetics (59\%) and in nonselected postprostatectomy patients ( $43 \%)$. In practice, other available phospodiesterase- 5 inhibitors also seem to have similar efficacy. In nonresponders, self-injection therapy with prostaglandin E1 (alprostadil) is often an effective treatment. Implantation of a penile prosthesis is the last resort $[17,18]$.

\section{Conclusion}

The neurologist should coordinate both the diagnosis and management of LUTD and SD in his/her patient, and a specialized nurse can provide much help both with assessment and counselling. Neurological departments, particularly those specialized in treating MS and movement disorder patients, should establish uroneurological expertise and should cooperate with an interested neurourologist, to whom complex cases should be referred.

\section{References}

1 Birder L, Chai T, Griffiths D, Grundy D, Thor K, Valentino R: Neural control; in Abrams P, Cardozo L, Khoury S, Wein A (eds): Incontinence. Paris, ICUD-EAU, 2013, chapter 10, pp 431-508.

-2 Stoehrer M, Blok B, Castro-Diaz D, ChartierKastler E, Del Popolo G, Kramer G, Pannek J, Radziszewski P, Wyndaele JJ: EAU Guidelines on Neurogenic Lower Urinary Tract Dysfunction. Eur Urol 2009;56:81-88.

3 Madersbacher H: The various types of neurogenic bladder dysfunction: an update of cur- rent therapeutic concepts. Paraplegia 1990; 28:217-229.

4 Basson R: The female sexual response. A different model. J Sex Marital Ther 2000;26:5165.

5 Gruenwald I: Male genital anatomy and physiology; in Porst H, Reisman Y (eds): The ESSM Syllabus of Sexual Medicine. Amsterdam, Medix Publishers, 2012, chapter 2, pp 30-41.

6 Cuzin B: Anatomy and physiology of female sexual organs; in Porst $\mathrm{H}$, Reisman Y (eds):
The ESSM Syllabus of Sexual Medicine. Amsterdam, Medix Publishers, 2012, chapter 3, pp 46-73.

7 Podnar S, Oblak C, Vodušek DB: Sexual function in men with cauda equina lesions: a clinical and electromyographic study. J Neurol Neurosurg Psychiatry 2002;73:715720 .

8 Fowler CJ, Panicker JN, Emmanuel A: Pelvic Organ Dysfunction in Neurological Disease. Cambridge, Cambridge University Press, 2010. 
9 Rotar M, Tršinar B, Kisner K, Barbič M, Sedlar S, Gruden J, Vodušek DB: Correlations between the ICIQ-UI short form and urodynamic diagnosis. Neurourol Urodyn 2009;28: 501-505.

10 Keleher C, Staskin D, Cherian P,Cotterrill N, Coyne K, Kopp Z, Symods T: Patient reported outcome assessment; in Abrams P, Cardozo L, Khoury S, Wein A (eds): Incontinence. Paris, ICUD-EAU, 2013, pp 431-508.

11 Reisman Y: Let's talk about sex: taking a sexual history; in Porst H, Reisman Y (eds): The ESSM Syllabus of Sexual Medicine. Amsterdam, Medix Publishers, 2012, chapter 10, pp 236-310.

12 Kalsi V, Fowler CJ: Therapy Insight: bladder dysfunction associated with multiple sclerosis. Nat Clin Pract Urol 2005;2:492-501.
13 Rosier R, Kuo HC, De Gennaro M, Kakizaki $\mathrm{H}$, Hashim H, Van Meel TD, Hobson PT: Urodynamic testing; in Abrams P, Cardozo L, Khoury S, Wein A (eds): Incontinence. Paris, ICUD-EAU, 2013, pp 431-508.

14 Tubaro A, Vodušek DB, Amarenco R, Doumouchtsis ST, DeLancey JOL, Fernando R, Khullar V, Puccini F, Podnar S: Imaging, neurophysiological testing and other tests; in Abrams P, Cardozo L, Khoury S, Wein A (eds): Incontinence. Paris, ICUD-EAU, 2013, pp 509-597.
15 Drake MJ, Apostolidis A, Emmanuel A, Gajewski J, Harrison SCW, Heesakkers J, Lemack G, Madersbacher H, Panicker J, Radzisewski P, Sakakibara R, Wyndaele JJ: Neurologic urinary and faecal incontinence; in Abrams $\mathrm{P}$, Cardozo L, Khoury S, Wein A (eds): Incontinence. Paris, ICUD-EAU, 2013, pp 827-954.

16 Bronner G, Vodušek DB: Management of sexual dysfunction in Parkinson's disease. Ther Adv Neurol Disord 2011;4:375-383.

17 Vodušek DB, Aminoff MJ: Sexual dysfunction in patients with neurological disorders; in Aminoff MJ (ed): Neurology and General Medicine, ed 4. Philadelphia, Churchill Livingstone Elsevier, 2008, chapter 34, pp 651-672.

18 Vodušek DB: Sexuelle Störungen aus der Sicht des Neurologen. Nervenarzt 2011;82:787802. 